\title{
Foam EOR performance in homogeneous porous media: simulation versus experiments
}

\author{
Ahmed Zoeir ${ }^{1} \cdot$ Mohammad Simjoo $^{1} \cdot$ Mohammad Chahardowli $^{1} \cdot$ Mojtaba Hosseini-Nasab ${ }^{2}$
}

Received: 23 July 2019 / Accepted: 27 January 2020 / Published online: 28 April 2020

(c) The Author(s) 2020

\begin{abstract}
In petroleum industry after primary production, associated gas released from separators or an inert gas from an external resource is injected into reservoir to push the oil toward production wells. The most important issue in injection process is that highly mobile fluids prefer to channel through porous media outgoing the oil phase. To overcome this problem, foam is injected into reservoir to increase sweep efficiency via reducing mobility ratio. Foam EOR performance in porous media depends on several factors including foam texture, media characteristics and injection scenarios. In this work, we used MATLAB software to numerically simulate foam core flooding and also to fit STARS parameters with data achieved from two previously performed foam injection experiments in Bentheimer core samples with lengths of 6.7" and 15.1". Then we used CMG software to outstretch foam EOR simulations to more realistic 2D and 3D geometries which were designed to study override issue in slant formations and also well patterns influence on foam EOR performance. Results show that foam EOR performance in slant pay zones with severe dip angles highly depends on foam strength, pay zone thickness and injection well location. Results also show that since foam always exhibits shear thinning characteristic, well patterns which use higher number of wider spread injection wells can aid to improve oil extraction efficiency during foam flooding.
\end{abstract}

Keywords Foam EOR $\cdot$ Bentheimer sandstone $\cdot$ CMG STARS $\cdot$ Well pattern $\cdot$ Slant formation

\section{Introduction}

In petroleum production industry whenever the reservoir pressure falls near saturation point, associated gas released in separators or an inert gas from an external resource is injected into reservoir due to two main reasons, firstly to maintain pressure and secondly to push the oil toward production wells. The most important issue during injection process is that highly mobile fluids prefer to channel through porous media outgoing oil phase.

To overcome this problem, foaming agent is co-injected with gaseous phase into reservoir to increase sweep efficiency via controlling the mobility ratio. In foam phase, gas pockets are separated from each other with a thin liquid layer which is called lamella. When the foam phase flows through porous

Ahmed Zoeir

ah.zoeir@sut.ac.ir

1 Faculty of Petroleum and Natural Gas Engineering, Sahand University of Technology, Tabriz, Iran

2 Department of Petroleum Engineering, Amirkabir University of Technology, Tehran, Iran media, this thin layer which has higher viscosity is in contact with internal solid surface of the porous media. Furthermore, shape change of large foam globules while passing through porous media throats applies momentum loss, therefore additional resistance during flow. All such microscopic effects make foam's apparent viscosity relatively much higher than those of original gas. Another issue in the gas injection process which is relevant to difference in fluid densities is gravity override. Using foaming agent can resolve this problem to some extent by reducing vertical transmissibility of gaseous phase while leaving density difference almost unchanged. Foam injection performance in porous media depends on several factors including foam texture, media characteristics, injection scenarios, etc. Several attempts were performed in the literature to investigate foam flow efficiency in various type porous media. Since foam rheology was not truly understood in 1980s and early years of 1990s, experimental studies were performed to investigate factors affecting foam flow in porous media. On this subject, several foam flooding experiments were carried out in transient rather than steady-state conditions at relatively high pressures in order to simulate reservoir conditions. Saturation profiles were drawn using 
high-energy electromagnetic equipment; thereafter, fractional flow theory was applied to extract relative permeability curves for foam phase. It was shown that liquid velocity linearly impacts pressure gradient within the core, while gas velocity which determines foam quality has small effects (Persoff et al. 1991). As time went by, fundamental concepts of foam flow in porous media including foaming agent compatibility with original gas like or as well as surfactant adsorption on various minerals were more precisely understood. Several various usages of gas foaming in upstream oil industry such as its applications in enhancing oil recovery as well as its usages in exploration technologies were also discussed (Schramm and Smit 1996). In the later years investigators tried to introduce representative models that describe foam phase behavior when flowing through porous media under various oil saturations, water salinities, etc. In this way theoretical studies were done to simulate previously performed foam injection experiments on carbonate core samples using steady-state foam models in one-dimensional environment. Problems like foam override were also studied using vertical geometrical models which express cross sections through a real field case study (Shrivastava et al. 1997). Pilot scale foam flooding application did not confine to the above example. In another case, gas foaming was applied in Snorre field located in North Sea to solve premature high problem within a production well. surfactant was injected into a high permeable layer to reduce by about 50\%. Such an application illustrated that generating a strong foam in an oil formation is not only achievable but also gives us several valuable profits when using as an improved oil recovery technique in field scale (Svorstol et al. 1997). In the way of developing foam models, investigators applied semi-empirical rather than empirical approaches to simulate foam flow in cross-sectional geometries. Essential parameters for each foam model were introduced; thereafter, several available approaches to calculate such factors were also discussed. It was shown that various empirical or semi-empirical methods can predict foam flow properties with acceptable accuracy (Kovscek 1998). Decrease in gravity override issue as an important consequence in foam application was studied in near-well region via numerical simulation using cylindrical rather than rectangular systems. It was illustrated that calculational factors like mesh size or orientation can affect predictions to some extent. It was also shown that tricks like nonuniform meshing can overcome simulation problems (Shi and Rossen 1998). In recent years, several developed foam flow models were added to reservoir simulator commercial software including along with simulators. In an investigation, two foam simulator software, namely in addition to was applied to fit foam model factors with experimental data in low rather than high foam quality regimes. Override problem was also studied in a vertical two-dimensional space using these simulators (Cheng et al. 2000). Influences of foaming agent concentration on foam phase rheology in an induced sandpack core sample was experimentally studied. with various AOS concentrations was applied to form foam with various strengths for injecting into core samples. Numerical simulations were then performed to fit foam model parameters with experimental data (Apaydin and Kovscek 2001). In some works fractional flow theory was used to analytically study foam injection process in one-dimensional space. Several sensitivity analyses were done to investigate impacts of foam quality, injection rate as well as foam strength on the process efficiency. It was shown that saturation profiles rather than differential pressures that came from analytical approach were in nice agreement with experimental data (Dholkawala et al. 2007). Further in this literature two various original gases were selected to perform foam injection experiments in granular porous media. To do this, along with at high pressure and temperature conditions was applied to form foam in media which was previously saturated with surfactant. It was shown that the pressure drops were always lower in foam flooding. while liquid recovery was higher in foam flooding (Farajzadeh et al. 2009). Foam flooding experiments in sandstone core samples were also addressed in an article. Then, an empirical foam approach from a common simulator was applied to express experimental data. Results illustrated relatively accurate predictions on pressure differentials with minor mismatch in entrance region (Chen et al. 2010). In another work researchers used Bentheimer core samples which were initially saturated with oleic phase to perform foam injection experiments. Inasmuch as opposite to foam, oleic phase has constant viscosity oil; relative permeability curves were fitted with pressure data from experiments using simple Darcy's linear equation. It seems in their work oil Rel Perm curves were underestimated to some extent (Heins et al. 2014). In another investigation wide range of experimental data was used to suit foam model factors. In their work fitting progress on each individual parameter was explained in detail. It was professed that their methods would apply to fit rather than Eclipse foam approaches without significant error (Boeije and Rossen 2015). A comparative study on foam coreflood experiments was made in which various foaming agent concentrations were used to form weak, intermediate as well as strong foams. Thereafter coreflood experiments were simulated using an implicit-texture model. It was shown that results that came out of numerical simulation were in nice agreement with experimental data (Jones et al. 2016). Puma-Flow is another software that was used to match empirical foam model coefficients with data extracted from previously performed foam injection experiments on a Bentheimer sandstone core sample. As an outcomes, two parameter sets relevant to two sets of core flooding experiments, with and without oleic phase, were wrote up (Hosseini-Nasab et al. 2018). Recently foam application in carbonate reservoirs was simulated using commercial software. factors were applied to model foam flow in fracture network rather than matrix units. Sensitivity analyses were 
then performed to investigate impacts of rock type, fracture network transmissibility, foam strength as well as injection rate, on efficiency of the foam oil gravity drainage mechanism (Zoeir et al. 2019).

In this paper, we numerically simulate foam injection experiments which were previously performed by one of the coauthors on two Bentheimer core samples with 6.7" and 15.1" lengths. We developed two codes in which use mass conservation law, Darcy's flow equation, capillary pressure relationship, to numerically simulate three-phase flow in one-dimensional porous media. The novelty of this study is that -type relative permeability curves relevant to each phase were extracted from fitting simulation results with experimental data. Then in order to unify foam phase relative permeability curves, we suit foam approach factors with simulation data from codes. Finally, these parameters were imported into software to outstretch our simulation results to more realistic and geometries. Foam override issue was then studied in several slant formation models with dip angle from $-45^{\circ}$ to $+45^{\circ}$. After that, four common patterns, namely normal five spot, inverted five spot, direct line drive as well as indirect line drive, were investigated to find out which one is more appropriate to suit non-Newtonian foam injection made up of gas plus aqueous solution.

\section{Methodology}

In this work, we perform several foam injection experiments in two Bentheimer core samples with 6.7" and 15.1" lengths. Nitrogen was co-injected with AOS surfactant into vertically placed short core, conversely into horizontally placed long one. Sensitivity analyses were then performed experimentally on foam injection rate and surfactant concentration while quality; therefore, texture is kept constant. After that, in order to provide an environment for simulating foam injection experiments, two codes were written in MATLAB to suit STARS model parameters in a way that satisfy experimental data of differential pressure achieved from Bentheimer sandstone cores. The calculated parameters were then used in CMG software to predict oil rate and recoveries from various foam injection scenarios in two-dimensional vertical cross-sectional models. These scenarios include foam injection with various qualities as follows: textures, foam injection at different rates, with and without surfactant pre-flush. Details about experiment steps, MATLAB coding and CMG simulations are presented further in this section. Two Bentheimer core samples with lengths of 6.7" and 15.1" were used in injection experiments.

Core properties and important materials used in experiments are listed in Table 1.

Cores were prepared in two various ways, first way in which cores were only saturated with formation water, and second
Table 1 Core properties and materials used

\begin{tabular}{lll}
\hline Core samples & Short & Long \\
\hline Length $(\mathrm{cm})$ & 17.0 & 38.5 \\
Direction & Vertical & Horizontal \\
Pore volume $\left(\mathrm{cm}^{3}\right)$ & $40.5 \pm 0.5$ & $90.5 \pm 0.5$ \\
Porosity $(\%)$ & $21.0 \pm 0.1$ & $20.0 \pm 0.1$ \\
Materials used & Name & Conc \\
Surfactant & AOS & $0.004 \%$ \\
Foaming gas & Nitrogen & $99.98 \%$ \\
Water salinity & $\mathrm{NaCl}$ & $0.5 \mathrm{M}$ \\
\hline
\end{tabular}

way in which cores were saturated with oleic phase. In order to do this, cores were initially evacuated with a vacuum pump; then, $\mathrm{CO}_{2}$ was injected into samples, and after that, water with formation salinity was injected to dissolve $\mathrm{CO}_{2}$. Details on experimental procedure and performed sensitivity analysis are listed in Table 2. When the cores were prepared, nitrogen gas and AOS (alpha olefin surfactant) as surface tension reducer were co-injected into cores to sweep formation water or oil from the cores. The short core was placed vertical while long core was set horizontally. Nitrogen gas flows through a regulator and then is co-injected with surfactant solution that comes from a liquid pump. Some valves were placed to conduct the flow to each individual core holder. Several pressure gauges were used to measure pressure drop along each core holder. Oil or water coming out from each experiment is collected in liquid collector while gas is conducted to a gas capsule. Schematic setup is shown in Fig. 1.

To perform foam injection experiments, two sensitivity analysis series were performed: sensitivities over foam strength, respectively, on surfactant concentration, and over injection rate. Both series were performed on short and long core samples and also in conditions with and without oleic phase. Experiments exclude sensitivity analysis on foam quality or other parameters like solution salinity. Since foam injection rates increase, two-phase velocities increase simultaneously to leave foam quality unchanged. As foaming agent concentration increases, more strong oil sweep efficiency is expected. In order to construct an environment to model foam injection experiments, we wrote two codes in MATLAB software for simulating multiphase flow with or without gravity for conditions in which core sample is kept vertical or horizontal, respectively. Saturation data came from CT scanning showing negligible variation in sections perpendicular to core axis; therefore, authors decided not to use $2 \mathrm{D}$ or $3 \mathrm{D}$ geometries in programming simulations.

In order to perform calculations, two geometries including vertical grids which represent short core, and horizontal grids which represent long core were defined. Then, we applied governing equations for multiphase flow in porous media including mass and momentum conservation equations. 
Table 2 Experimental procedure steps

Pre experiment procedure

\begin{tabular}{ll}
\hline Water saturated & Oil Saturated \\
Evacuated & Evacuated \\
$\mathrm{CO}_{2}$ injection & Brine injection \\
Brine injection & Oil saturation \\
\hline
\end{tabular}

Performed sensitivity analyses

\begin{tabular}{lrrrrr}
\hline \multicolumn{2}{l}{ Surfactant Conc (\%) } & \multicolumn{4}{l}{ Injection rate (ft/D) } \\
0.10 & 0.50 & 1.00 & 1.40 & 2.80 & 5.60 \\
\hline
\end{tabular}

$-\frac{\partial\left(\rho_{i} u_{i, x}\right)}{\partial x}-q_{i}=\frac{\partial\left(\emptyset \rho_{i} S_{i}\right)}{\partial t} \quad i=f, o, w$

$-\frac{\partial\left(\rho_{i} u_{i, z}\right)}{\partial z}-q_{i}=\frac{\partial\left(\emptyset \rho_{i} S_{i}\right)}{\partial t} \quad i=f, o, w$

$u_{i, x}=-\frac{k k_{r i}}{\mu_{i}} \frac{\partial P_{i}}{\partial x} \quad i=f, o, w$

$u_{i, z}=-\frac{k k_{r i}}{\mu_{i}}\left(\frac{\partial P_{i}}{\partial z}+g\right) \quad i=f, o, w$

in which three phases of foam, oil and water are mobile. Adjusting momentum equation into mass conservation equation gives.

$\frac{\partial}{\partial x}\left(\frac{k k_{r i}}{\mu_{i} B_{i}} \frac{\partial P_{i}}{\partial x}\right)-q_{i}=\frac{\partial}{\partial t}\left(\frac{\partial\left(\emptyset S_{i}\right)}{B_{i}}\right) \quad i=f, o, w$

$\frac{\partial}{\partial z}\left(\frac{k k_{r i}}{\mu_{i} B_{i}}\left(\frac{\partial P_{i}}{\partial z}+g\right)\right)-q_{i}=\frac{\partial}{\partial t}\left(\frac{\partial\left(\emptyset S_{i}\right)}{B_{i}}\right) \quad i=f, o, w$
In order to solve these equations numerically, single dimension discretization was performed; thereafter, required parameters like operating conditions, rock properties, injection scenarios were adjusted. Finally, equations were solved using IMPES method which applies implicit solution for pressure and explicit solution for saturation calculations.

One must notice to three main concepts which affect multiphase flow in porous media, namely capillary, viscous and gravitational forces. Porous media desires to absorb wetting phase to an extent which depends on capillary force which itself depends on saturation values in each special position within the media. In this work, since no information is available on capillary force, authors decided to exclude this concept in calculations. Another force which obviously plays an important role in flooding experiments is viscous force which came from applying external pressure difference. Another force affecting flooding experiments in core samples that are held non horizontal is the gravitational force. In our study, wherever foam injection experiments took place in horizontally placed long core, with or without oleic phase, gravity has no impact on multiphase flow in porous media. Conversely in cases in which flooding took place in vertical short core, gravity force opposes displacement process. Magnitude of the driving forces is compared for all our series of experiments as shown in Table 3.

In our case studies, since viscous force is about hundred times larger than gravity driving force, viscous dominant flow took place in vertical core flooding rather than horizontal flooding experiment. In cases in which foam injection was simulated in two-dimensional space using CMG software, injecting flow rates were also large enough to provide viscous dominant flow. Two main experiment series, with and without oleic phase, were modeled separately. In order to estimate water relative permeability curve, experiments without oleic phase were used. To do this, LET-type curves with three independent parameters were presumed to present
Fig. 1 Schematic setup previously used for foam injection experiments

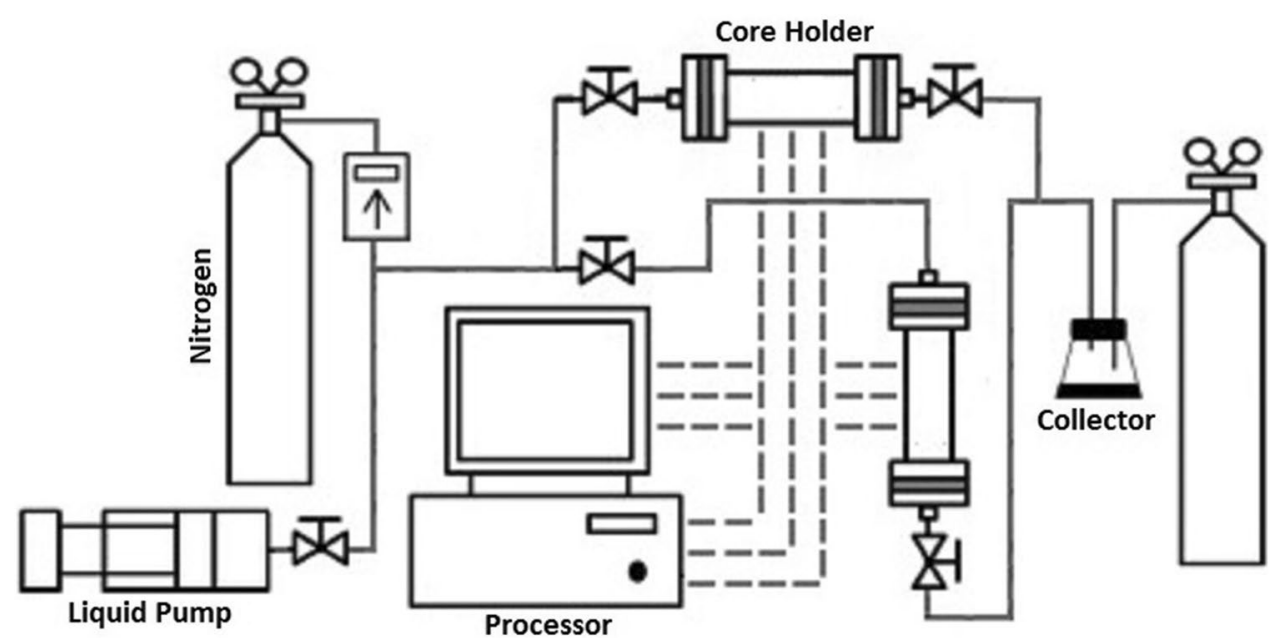


Table 3 Driving force comparison

\begin{tabular}{llllll}
\hline Sample & \multicolumn{2}{l}{ Viscous (psi) } & & \multicolumn{2}{l}{ Gravitational (psi) } \\
\cline { 2 - 3 } \cline { 5 - 6 } & \multicolumn{2}{l}{ Displaced fluid } & & \\
\cline { 2 - 3 } & Oil & Water & & Oil & Water \\
\hline Vertical core & 65.3 & 45.3 & 0.19 & 0.27 \\
Horizontal core & 148.2 & 113.4 & & 0.0 & 0.0 \\
\hline
\end{tabular}

Rel Perm rather than Corey type in which two parameters exist.

$$
\begin{aligned}
& K_{r w}=\frac{K_{r w}^{o} S_{w n}^{L_{w}}}{S_{w n}^{L_{w}}+E_{w}\left(1-S_{w n}\right)^{T_{w}}} \\
& K_{r o}=\frac{\left(1-S_{w n}\right)^{L_{o}}}{\left(1-S_{w n}\right)^{L_{o}}+E_{o} S_{w n}^{T_{o}}}
\end{aligned}
$$

To fit LET-type curve parameters with pressure drops, recorded using differential gauges, also with saturation profiles monitored using CT scan, a trial-and-error procedure was designed. Iteration process starts with an estimation of LET factors assuming water-wet condition for Bentheimer sandstone. Then, the parameters undergo some independent sensitivity analyses to find out their optimum value in which least integrated square error does appear among estimated and experimental saturation data.

Converse to water phase, Rel Perm curves for foam and oil phases were extracted from data on second series of experiments in which oleic phase was present. According to Stone's theory, it was assumed that the water Rel Perm curve is same; nevertheless, oil phase is present or not. Similar trial-and-error method was again used to fit simulated saturation results with those of experiments. Six LET-type curve factors were optimized to express Rel Perm curves of foam and oil, respectively.

Since several experiments with various AOS concentrations, different injection rates were performed on short and long core samples; foam's Rel Perm curves differ case by case. Foam strength increases as AOS concentration increase makes Rel Perm curves to deviate more from original nitrogen gas. In addition to that, since foam is a nonNewtonian fluid, its mobility is dependent on linear velocity, hence injection rate. All above notifications inform us that we should expect various Rel Perm curves for foam phase in each individual experiment. In order to unify such an effect, we developed codes in MATLAB to fit CMG's foam approach, STARS parameters with estimated Rel Perm curves. STARS model considers foam's viscosity same as gas and applies all changes in mobility, only in Rel Perm correction factor. Simplified STARS model which is used in CMG 2006 is shown here.

$k_{r}^{f}=k_{r}^{g} * \mathrm{FM}$

$\mathrm{FM}=\frac{1}{1+F_{\max } * F_{\mathrm{AOS}} * F_{\text {cap }} * F_{\text {water }} * F_{\text {oil }}}$

in which $F_{\max }$ represents maximum possible change in gas Rel Perm due to the presence of foaming agent. $F_{\mathrm{AOS}}, F_{\text {cap }}$, $F_{\text {water }}, F_{\text {oil }}$ are parameters that represent AOS impact, nonNewtonian effect, water and oil phase influences, respectively. All these factors express foam's behavior with some power law function which is almost fully described via two independent sub-parameters. In order to determine STARS model factors, we used each sensitivity analysis series of experiments separately. Experiments in which AOS surfactant concentration was changed were used for $F_{\mathrm{AOS}}$ calculation, and those in which injection rate was varied were used to calculate $F_{\text {cap }}$, etc. Graphical user interface window relevant to the developed MATLAB codes is presented in Fig. 2.

Finally to extend 1D simulation results to more realistic $2 \mathrm{D}$ vertical cross-sectional models and 3D EOR patterns, optimized STARS parameters were imported into CMG software. Foam override as a phenomenon which only appears in multi-dimensional space was studied via simulating foam injection in some vertical cross-sectional geometries with various dip angles in which foam was injected from one side to push the oil to production well. Foam flooding with different strengths into various angle slant formations was simulated in CMG to investigate override issue wherever foam was used in field scale. In addition to that, 3D simulations were performed to estimate oil sweep efficiencies in some various standard EOR patterns including normal five spot, inverted five spot, direct line drive and indirect line drive. Swept areas and foam's average flow velocities for each pattern after several years of production were reported; thereafter, we discussed which one was more profitable. One can expect simulations to stretch to situations with extrapolated AOS concentrations, conditions with different initial oil saturations and various injection rates. We should also notice that the results cannot be extended to other rock types rather than Bentheimer or other surfactants rather than AOS.

\section{Results and discussions}

In this section, firstly we present the appraised mutiphase relative permeabilities which were extracted from fitting $1 D$ multiphase flow formulas with experimental data, in terms of LET-type curve parameters. Then, we used our written MATLAB codes to reprint unsteady state saturation curves

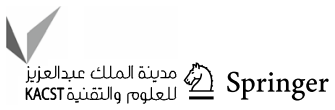




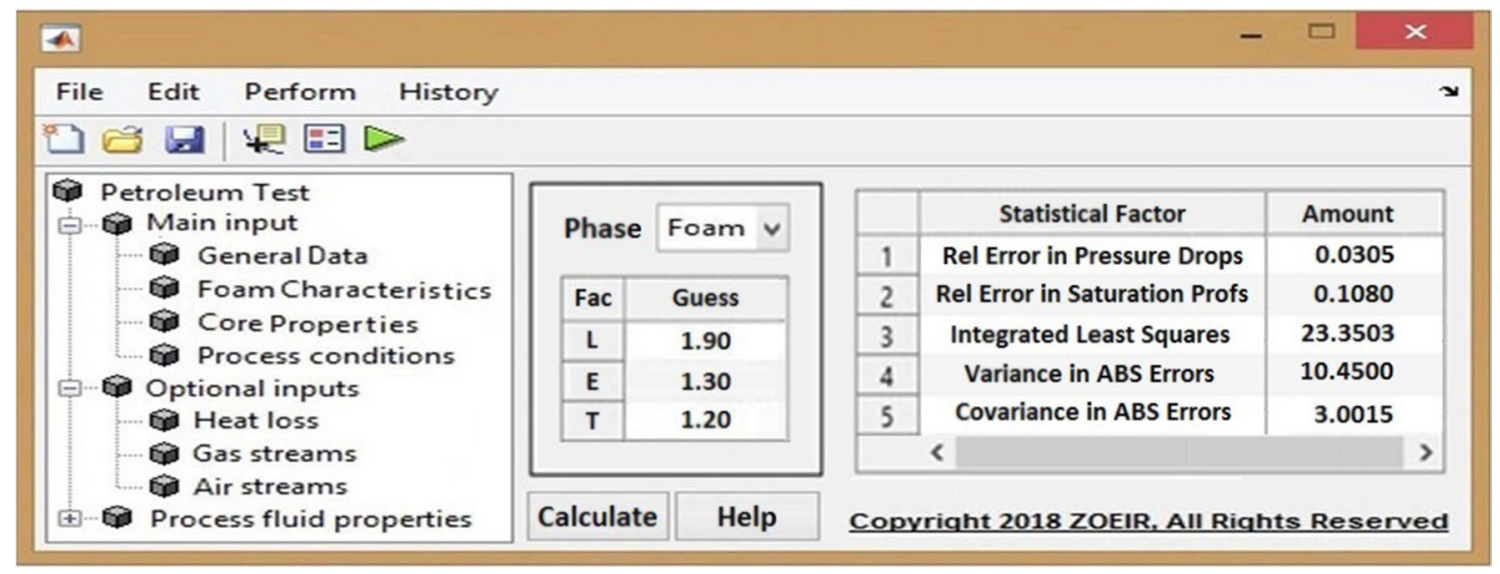

Fig. 2 An image of the developed MATLAB GUI code to calculate Rel Perms and STARS parameters

in order to verify and validate our calculations with curves extracted directly from micro-CT scanning method. After that, we used STARS foam model to unify multiple Rel Perm curves drew for various conditions of AOS concentration and also injection rate. Further in this section, we used CMG 2015 software to extend 1D simulations to 2D vertical cross section and some 3D standard EOR patterns. One can use three datasets to fit core flooding experimental data with simulations, namely saturation curves recorded using CT scan, differential pressures measured along core sample or recovery curves drew after experiment. Using pressure data which represent flow resistance in various locations along sample during flooding is more precise than using recovery data. But in cases in which saturation distribution data are available from CT scanning, just like our case, fitting Rel Perm curves with phase saturations are wiser choice. In order to estimate LET parameters that fully describe mutiphase relative permeabilities, three-phase saturation data were imported into the written MATLAB codes. Saturation profiles came from core flooding experiments without oleic phase were utilized in calculating aqueous phase Rel Perm while those relevant to core flooding experiments with oleic phase were used to estimate oil Rel Perm. Firstly, typical LET-type factors were initially estimated; thereafter, an iteration process was started to find optimum parameters. In each step, saturation curves came from simulations and were drew against recorded experimental data and absolute errors were recorded. Regarding that, $L$ factor controls flow characteristics wherever phase saturation is low; conversely, $T$ influences flow at high-phase saturations, and iteration process focuses on fitting special parameters in such circumstances. Iteration procedure continues until saturation profiles that came from modeling show nice match with experimental data. Since LET-type Rel Perm curves express rock-fluid interaction with three independent factors, namely $L, E$ and $T$ which regulate lower, middle and upper part of the diagram, respectively, one can more truly explain fluid's flow in porous media near their irreducible saturation.

Experience using LET correlation indicates that reasonable ranges for parameters are $L \geq 0.1, E>0.1, T \geq 0.1$. Since each experiment was performed using especial foam phase characteristics, including AOS concentration or injection rate, different Rel Perm curves, various LET factors were extracted for foam phase in each experiment. Optimum values of LET parameters, for each of the three phases, are shown in Table 4.

Relative permeabilities relevant to water phase were extracted from experiments which were performed on 6.7" and 15.1" length cores without oleic phase. Authors supposed that the Stone's law is dominant; therefore, waterphase Rel Perm remains unchanged when oil is drained into porous media. Conversely, foam- and oil-phase Rel Perm curves were extracted from second experiment series in which oleic phase was present. Factor T controls top part within the Rel Perm curve while parameters $L$ and $E$ are relevant to lower and middle parts, respectively. Since an
Table 4 Relative permeabilities LET-type curve parameters for multiphases of foam, oil and water

\begin{tabular}{lllllllll}
\hline Curve factors & Oleic phase & Formation brine & \multicolumn{6}{l}{ Foam with various injection conditions } \\
\cline { 4 - 8 } & & & $0.1 \%$ & $0.5 \%$ & $1.0 \%$ & $1.4 \mathrm{ft} / \mathrm{D}$ & $2.8 \mathrm{ft} / \mathrm{D}$ & $5.6 \mathrm{ft} / \mathrm{D}$ \\
\hline$L$ & 1.90 & 1.70 & 2.10 & 2.00 & 1.90 & 2.10 & 2.20 & 2.30 \\
$E$ & 1.30 & 1.10 & 1.50 & 1.40 & 1.30 & 1.50 & 1.60 & 1.70 \\
$T$ & 1.00 & 1.90 & 1.40 & 1.30 & 1.20 & 1.40 & 1.50 & 1.60 \\
\hline
\end{tabular}


increase in alpha olefin sulfonate concentration in aqueous solution forms stronger foam with lower mobility ratio, its viscosity deviates more from original gas viscosity. Since STARS model considers all mobility changes only in foam phase Rel Perm, increasing surfactant concentration makes Rel Perm to decrease larger value from nitrogen gas Rel Perm curve. This concept is shown in calculated optimum LET factors for cases in which AOS concentrations vary. As surfactant concentration increases from 0.1 to $1.0 \mathrm{wt} \%$, all three factors tend to decrease making foam phase less mobile. This decrease is about $10-15 \%$ relatively against $1.0 \mathrm{wt} \%$ increase in AOS concentration. Since foam is a non-Newtonian fluid, its viscosity decreases as flow velocity increases causing less mobility control during injection. As injection linear velocity increases from 1.4 to $5.6 \mathrm{ft} / \mathrm{D}$, estimated Rel Perm for foam phase shows almost $10 \%$ increase in transmissibilities which show its shear thinning behavior.

In the following, to verify MATLAB simulations with experimental data we drew saturation profiles against time for foam injection into long core as shown in Fig. 3. Left photograph shows dynamic micro-CT images during foam flooding while the left diagram presents saturation profiles. Colorless diagrams show experimental data while colorful ones are extracted from MATLAB codings.

During foam injection into long Bentheimer core sample which its dynamic micro-CT scans are shown above, water saturation decreases from $100 \%$ to its residual saturation which is about $40 \%$, as foam injection time proceeds. Breakthrough time in this experiment shows amount of $0.68 \mathrm{PV}$ after which gas is produced at the end. As shown in rightside graph, saturation profiles which were predicted using MATLAB codes are in good agreement with experimental data extracted from micro-CT images.

Conversely in vertical short core sample in which foam was injected from top, gravity effect was turned on in MATLAB simulations. Breakthrough time in gravity stable flooding experiment shows lower absolute value and was divided by core length showing larger value. Main reason to this is that here highly viscous oil phase was added to core sample while in the previous experiment core was initially saturated with only low viscous water. Dynamic micro-CT scanning images during foam injection into short Bentheimer core sample are shown in Fig. 4. Pressure drop along core sample, mobility reduction factor and oil recovery versus time are parameters which are shown in graphs. When foam penetrates into the core sample replacing oil, overall pressure drop increases to approximately a plateau and then changes very slowly. Conversely, oil recoveries accelerate in early times and increase more smoothly in late times.

This probably means that in early times foam was displacing mainly low viscous water while in the late times, it mainly sweeps high viscous oil. Since AOS concentration increases from 0.5 to $1.0 \%$, MRF curves, therefore pressure drops, increase about $50 \%$ which shows high dependency of the mobility control on foam strength. In all diagrams, MATLAB simulation results show very good agreement with the measured data. This agreement verifies that the estimated LET Rel Perm factors are nearly true. While oleic phase Rel Perm just like aqueous phase is almost constant in all our experiments, foam phase Rel Perm is dependent on AOS concentration, injection rate, oil saturation, etc. In order to unify all Rel Perm curves relevant to foam phase, we applied CMG's foam approach STARS model to describe all its characteristics including impact of presence of oleic phase, non-Newtonian effect, viscosity change with surfactant concentration, etc. To do this, another code wrote to suit STARS parameters with LET Rel Perm functions. Each sensitivity analysis series of experiments was applied to find its relevant STARS factor. For instance, experiments in which surfactant concentration was varied were used to set $F_{\mathrm{AOS}}$. Experiments in which injection rates were changed were used to calculate $F_{\text {cap }}$, etc. STARS parameters are power law functions which consist of two or three independent sub-parameters. These functions were optimized, and resultant factors are listed in Table 5.
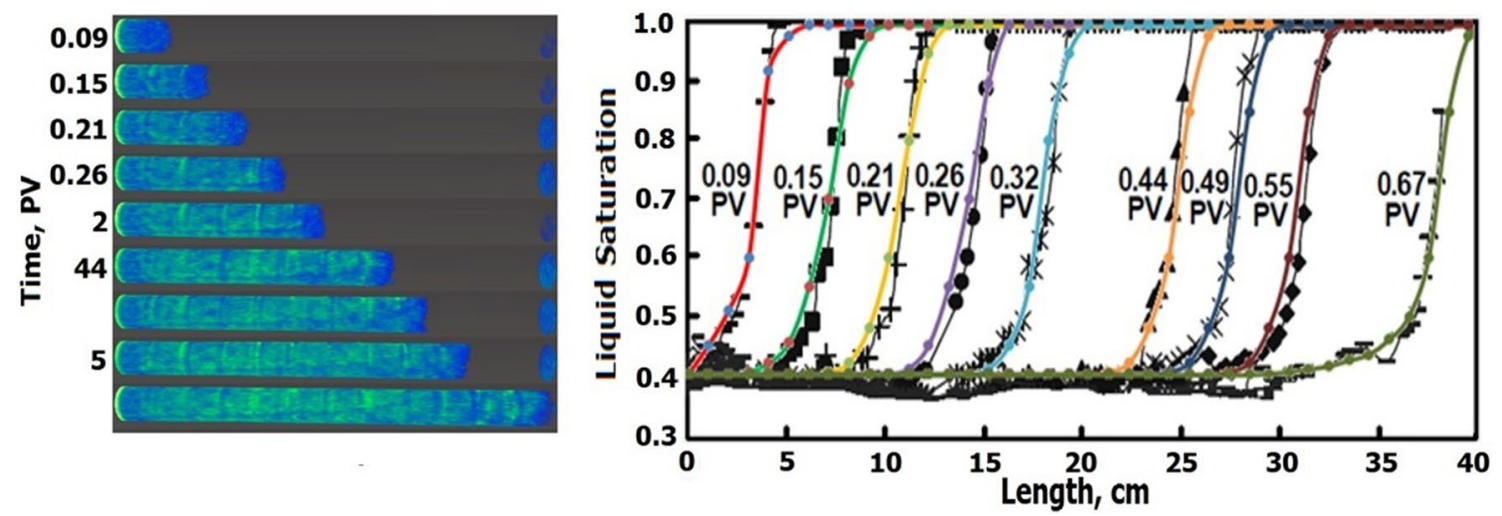

Fig. 3 Dynamic CT images (left) and saturation profiles for foam injection in long core (right) 

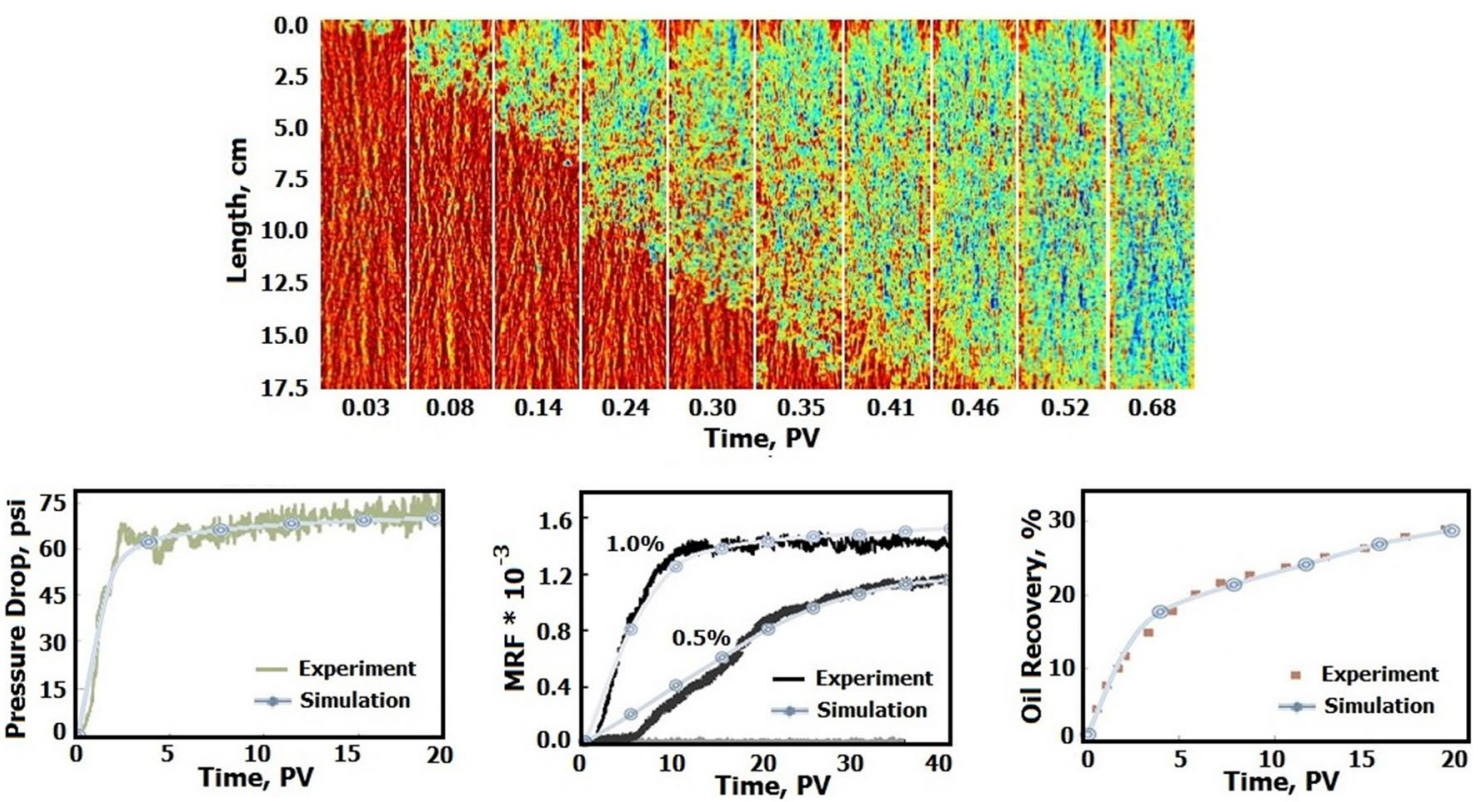

Fig. 4 Dynamic CT images (top), pressure drop, MRFs and oil recoveries for foam injection in short core

Outcome STARS parameters came from fitting simulations with saturation data from short and long core foam flooding experiments showing little difference. Since it was previously shown that the viscous force prevails in multiphase flow in conditions defined in this study, such a distinction may come from capillary end effect. Both core samples are Bentheimer sandstones with the similar properties except their lengths. It seems that longer core exhibits less undesired capillary end effect due to its larger size.

In the following, to outstretch calculations to more realistic 2D and 3D geometries, CMG 2015 software is used to investigate foam application issues whenever is used in field scale. Two main differences that appear during immiscible displacement simulation in 2D vertical porous media models rather than 1D geometries are, firstly, gravity force influences and, secondly, depth-dependent heterogeneity. Since our experiments were performed only on Bentheimer sandstone core samples and yet our MATLAB simulations estimate Rel Perm curves for this especial rock type, we cannot outstretch our calculations to heterogeneous media which contain other rock types. Instead, we can investigate impact of gravity override in reservoirs with various dip angles. To do this, we applied STARS foam approach to simulate several 2D vertical cross sections within inclined reservoirs. Dip angles range from $-45^{\circ}$ where foam was flowing downward to $+45^{\circ}$ in which foam was flowing upward, stepwise with $15^{\circ}$ step size. Two various states, thin with $100 \mathrm{ft}$ and thick with $300 \mathrm{ft}$ heights, were supposed to present pay zone thicknesses. Factors which were extracted from MATLAB codes to suit STARS model were used directly in CMG 2015 software to define foam characteristics. Two AOS concentration values, $1 \%$ and $3 \%$, therefore two different foam

Table 5 STARS foam formula parameters fitted on 1D simulation results

\begin{tabular}{|c|c|c|c|c|c|c|c|c|c|}
\hline \multirow{2}{*}{$\begin{array}{l}\text { With oil } \\
\text { Short Core }\end{array}$} & \multicolumn{2}{|c|}{$F_{\text {surf }}$ family } & \multicolumn{2}{|c|}{$F_{\text {cap }}$ family } & \multicolumn{2}{|c|}{$F_{\text {water }}$ family } & \multicolumn{3}{|c|}{$F_{\text {oil }}$ family } \\
\hline & epsurf & fmsurf & epcap & fmcap & epdry & fmdry & epoil & floil & fmoil \\
\hline & 0.65 & 0.35 & 0.25 & $1 e-7$ & 135 & 0.15 & 0.35 & 0.05 & 0.45 \\
\hline \multirow[t]{2}{*}{ Long Core } & epsurf & fmsurf & epcap & fmcap & epdry & fmdry & epoil & floil & fmoil \\
\hline & 0.60 & 0.35 & 0.20 & $1 e-7$ & 145 & 0.15 & 0.30 & 0.05 & 0.40 \\
\hline Without oil & \multicolumn{2}{|c|}{$F_{\text {surf }}$ family } & & \multicolumn{3}{|c|}{$F_{\text {cap }}$ family } & \multicolumn{3}{|c|}{$F_{\text {water }}$ family } \\
\hline \multirow[t]{2}{*}{ Short core } & \multicolumn{2}{|c|}{ epsurf } & fmsurf & \multicolumn{2}{|c|}{ epcap } & fmcap & \multicolumn{2}{|c|}{ epdry } & fmdry \\
\hline & \multicolumn{2}{|c|}{1.45} & 0.35 & \multicolumn{2}{|c|}{1.05} & $1 e-7$ & \multicolumn{2}{|c|}{950} & 0.15 \\
\hline \multirow[t]{2}{*}{ Long core } & \multicolumn{2}{|c|}{ epsurf } & fmsurf & \multicolumn{2}{|c|}{ epcap } & fmcap & \multicolumn{2}{|c|}{ epdry } & fmdry \\
\hline & \multicolumn{2}{|c|}{1.35} & 0.15 & \multicolumn{2}{|c|}{1.15} & $1 \mathrm{e}-7$ & \multicolumn{2}{|c|}{1050} & 0.15 \\
\hline
\end{tabular}


strengths, were assumed in simulations. Breakthrough times and recoveries after 10 years were presumed to represent sweep efficiency in foam injection processes. Simulation results are shown in Table 6 . In the cases with severe negative dip angles in which foam phase was injected from top, sweep efficiencies, therefore recovery factors, were high. Breakthrough times shorten four or five times wherever dip angles increase to positive values.

In thick pay zones, gravity effect enlarges making override issue more problematic during foam injection process. Conversely, whenever foam strength increases with dissolving more AOS surfactant, oil sweep efficiency increases significantly weakening override issue. Thus, to achieve higher sweep efficiency when applying foam EOR, using stronger foam is demanded especially in cases in which foam is flowing upward.

Finally, in order to extend simulation results to 3D geometries, we defined four more common EOR patterns in CMG software, namely normal five spot, inverted five spot, direct line drive and indirect line drive. Then, foam injection process was simulated in each geometry for 15 years; thereafter, factors like swept area, oil recoveries in various years, etc., were used for comparison as shown in Table 7. The most successful EOR pattern with highest recoveries was normal five spot while second place was assigned to direct line drive. Worst pattern which gave least recovery values was inverted five spot in which foam was injected in only one central well.

In order to explain such a conclusion, one must refer to foam's non-Newtonian characteristic. Since foam viscosity, therefore pressure drop along flow direction, increases wherever foam's average velocity is low, in cases in which more wells were used to inject fixed volume of foam, recoveries show higher values. Conversely in EOR patterns in which less injection wells exist, higher average velocities cause lower foam viscosity which increases the chance of channeling or fingering leading to lower oil sweep efficiencies.

Authors conclude that firstly, optimal well layout, therefore EOR pattern depends on the injecting fluid characteristics in addition to porous media properties. Secondly, wherever fixed volume of foam is injected into higher number of well with more spread layout, EOR process efficiency can increase to some extent.

\section{Conclusions}

Several key findings of the present paper can be summarized as follows:

(1) Using LET-type Rel Perm curves rather than Corey type, to fit simulation results with coreflooding data, gives us smaller relative errors. While water Rel Perm curve is almost constant regardless of the presence or absence of oleic phase, foam's Rel Perm is not. An advanced foam model like STARS is always needed to fully describe foam's flowing properties.

(2) Fitting STARS model parameters with using short core sample can cause errors. Since capillary end effect

Table 6 Foam's sweep efficiency and breakthrough times in various dip angles and pay zone thicknesses

\begin{tabular}{|c|c|c|c|c|c|c|c|c|c|}
\hline Dip angle & & Efficiency factor & $-45^{\circ}$ & $-30^{\circ}$ & $-15^{\circ}$ & $0^{\circ}$ & $15^{\circ}$ & $30^{\circ}$ & $45^{\circ}$ \\
\hline \multirow[t]{4}{*}{ Strong foam AOS $3 \%$} & \multirow[t]{2}{*}{ Thin $100 \mathrm{ft}$} & Breakthrough time in years & 13.0 & 11.4 & 9.8 & 8.0 & 7.3 & 5.3 & 3.5 \\
\hline & & Oil recovery after 10 years & $48 \%$ & $42 \%$ & $39 \%$ & $33 \%$ & $28 \%$ & $23 \%$ & $19 \%$ \\
\hline & \multirow[t]{2}{*}{ Thick $300 \mathrm{ft}$} & Breakthrough time in years & 14.8 & 12.5 & 10.0 & 7.7 & 7.4 & 5.5 & 3.6 \\
\hline & & Oil recovery after 10 years & $55 \%$ & $43 \%$ & $36 \%$ & $28 \%$ & $25 \%$ & $22 \%$ & $18 \%$ \\
\hline \multirow[t]{4}{*}{ Weak foam AOS $1 \%$} & \multirow[t]{2}{*}{ Thin $100 \mathrm{ft}$} & Breakthrough time in years & 10.3 & 9.8 & 8.0 & 5.0 & 3.2 & 1.8 & 0.8 \\
\hline & & Oil recovery after 10 years & $30 \%$ & $25 \%$ & $21 \%$ & $18 \%$ & $15 \%$ & $12 \%$ & $8 \%$ \\
\hline & \multirow[t]{2}{*}{ Thick $300 \mathrm{ft}$} & Breakthrough time in years & 11.6 & 10.3 & 8.8 & 4.6 & 3.0 & 1.7 & 0.7 \\
\hline & & Oil recovery after 10 years & $38 \%$ & $29 \%$ & $23 \%$ & $15 \%$ & $13 \%$ & $10 \%$ & $7 \%$ \\
\hline
\end{tabular}

Table 7 Various EOR patterns and their resultant swept areas and oil recoveries

\begin{tabular}{|c|c|c|c|c|c|c|c|c|c|}
\hline \multirow[t]{2}{*}{ EOR patterns } & \multicolumn{2}{|c|}{ Wells } & \multicolumn{3}{|c|}{ Swept area (acres) } & \multirow[t]{2}{*}{ Average velocity } & \multicolumn{3}{|l|}{ Oil recovery } \\
\hline & Inj & Pro & 5 year & 10 year & 15 year & & 5 years $(\%)$ & 10 years $(\%)$ & 15 years $(\%)$ \\
\hline Normal five spot & 4 & 1 & 630 & 950 & 1150 & $2.0 \mathrm{ft} / \mathrm{D}$ & 35 & 42 & 45 \\
\hline Inverted five spot & 1 & 4 & 750 & 1080 & 1240 & $3.0 \mathrm{ft} / \mathrm{D}$ & 27 & 34 & 37 \\
\hline Direct line drive & 2 & 2 & 690 & 1020 & 1200 & $2.5 \mathrm{ft} / \mathrm{D}$ & 32 & 38 & 42 \\
\hline Indirect line drive & 1 & 2 & 720 & 1050 & 1230 & $3.0 \mathrm{ft} / \mathrm{D}$ & 30 & 35 & 40 \\
\hline
\end{tabular}


always influences core flooding results especially in relatively low injection flow rates, it is more reasonable to use results from longer cores for fitting processes.

(3) Foam EOR performance in slant pay zones with severe dip angles highly depends on foam strength, pay zone thickness and injection well location. While foaming can significantly decrease override issue within the gas injection process, it can still reduce oil sweep efficiency by about $15 \%$.

(4) Optimal EOR pattern differs case per case depending on injecting and displaced fluid characteristics. In our simulation scenario with its especial rock type and materials used, normal five spot pattern shows highest performance. One can generally advice that if foam is injected into media in a way that exhibits lowest possible linear velocity, process efficiency increases.

Open Access This article is licensed under a Creative Commons Attribution 4.0 International License, which permits use, sharing, adaptation, distribution and reproduction in any medium or format, as long as you give appropriate credit to the original author(s) and the source, provide a link to the Creative Commons licence, and indicate if changes were made. The images or other third party material in this article are included in the article's Creative Commons licence, unless indicated otherwise in a credit line to the material. If material is not included in the article's Creative Commons licence and your intended use is not permitted by statutory regulation or exceeds the permitted use, you will need to obtain permission directly from the copyright holder. To view a copy of this licence, visit http://creativecommons.org/licenses/by/4.0/.

\section{References}

Apaydin OG, Kovscek AR (2001) Transient foam flow in homogeneous porous media: surfactant concentration and capillary end effects. In: SPE improved oil recovery symposium Tulsa

Boeije CS, Rossen WR (2015) Fitting foam-simulation-model parameters to data: I. Coinjection of gas and liquid. SPE Reserv Eval Eng 18:264-272

Chen Q, Gerritsen MG, Kovscek AR (2010) Modeling foam displacement with the local-equilibrium approximation: theory and experimental verification. SPE Annual Technical Conference and Exhibition Denver Canada

Cheng L, Reme AB, Rossen WR, (2000) Simulating foam processes at high and low foam qualities. In: SPE improved oil recovery symposium Oklahoma

Dholkawala ZF, Sarma HK, Kam SI (2007) Application of fractional flow theory to foams in porous media. J Pet Sci Eng 57:152-165

Farajzadeh R, Andrianov A, Bruining J (2009) Comparative study of $\mathrm{CO}_{2}$ and $\mathrm{N}_{2}$ foams in porous media at low and high pressuretemperatures. Ind Eng Chem Res 48(9):4542-4552

Heins R, Simjoo M, Zitha PLJ (2014) Oil relative permeability during enhanced oil recovery by foam flooding. In: SPE annual technical conference and exhibition Netherland

Hosseini-Nasab SM, Douarche F, Simjoo M (2018) Numerical simulation of foam flooding in porous media in the absence and presence of oleic phase. Fuel 225:655-662

Jones SA, Laskaris G, Farajzadeh R (2016) Effect of surfactant concentration on foam: from coreflood experiments to implicit texture foam model parameters. J Ind Eng Chem 37:268-276

Kovscek AR (1998) Reservoir simulation of foam displacement processes. In: 7th UNITAR international conference on heavy crudes and tar sands, pp 27-31

Persoff P, Radke CJ, Pruess K (1991) A laboratory investigation of foam flow in sandstone at elevated pressure. In: SPE reservoir engineering, pp 185-192

Schramm LL, Smit DH (1996) Foams: fundamentals and applications in the petroleum industry, vol 10. American Chemical Society, Washington

Shi JX, Rossen WR (1998) Simulation of gravity override in foam processes in porous media. SPE Reserv Eng 1:148-154

Shrivastava VK, Belgrave JDM, Singhal AK (1997) Numerical simulation of foam flooding for sweep improvement. J Can Pet Technol $38: 45$

Svorstol I, Blaker T, Tham MJ (1997) A production well pilot in The North Sea Snorre field-application of foam to control gas breakthrough. In: International symposium of the society of core analysts in Austin Texas USA

Zoeir A, Chahardowli M, Simjoo M (2019) Foam application in fractured carbonate reservoirs: a simulation study. Iran J Oil Gas Sci Technol 4:18-34

Publisher's Note Springer Nature remains neutral with regard to jurisdictional claims in published maps and institutional affiliations. 